\title{
COMPACT SUBGROUPS OF LIE GROUPS AND LOCALLY COMPACT GROUPS
}

\author{
KARL H. HOFMANN AND CHRISTIAN TERP
}

(Communicated by Roe Goodman)

\begin{abstract}
We show that the set of compact subgroups in a connected Lie group is inductive. In fact, a locally compact group $G$ has the inductivity property for compact subgroups if and only if the factor group $G / G_{0}$ modulo the identity component has it.
\end{abstract}

\section{THE BASIC IDEAS}

For a topological group $G$ let $\mathscr{C}_{G}$ denote the set of compact subgroups of $G$ partially ordered by inclusion $\subseteq$. In the structure theory of Lie groups the following facts are frequently used: Suppose that $G$ is a Lie group with finitely many components.

Theorem A. Every member of $\mathscr{C}_{G}$ is contained in a maximal member of $\mathscr{C}_{G}$.

Theorem B. Two maximal members of $\mathscr{C}_{G}$ are conjugate.

Theorem C. For any maximal member $K \in \mathscr{C}_{G}$, there is a submanifold $E$ diffeomorphic to $\mathbb{R}^{n}$ for some $n=0,1,2, \ldots$ such that $(k, e) \mapsto k e: K \times E \rightarrow$ $G$ is a diffeomorphism.

Theorems A and B constitute analogs of Sylow's Theorems for compact subgroups. Textbooks tend to shun these theorems because of their difficulty-one presumes. Not even Bourbaki [2] presents them in his first nine chapters. A canonical source is the book by Hochschild [7].

Let $G_{0}$ denote the identity component of a topological group $G$. The theorems above generalize at once to locally compact groups $G$ with compact factor group $G / G_{0}$ as soon as one accepts the fact that these groups are projective limits of Lie groups [11, p. 175; 4]. This generalisation is much easier than the proof of any of Theorems A, B, and C. All the information accumulated on Lie groups has not helped to provide any shortcuts. A fresh look may be useful.

Clearly, via Zorn's Lemma, Theorem A is a direct consequence of

Received by the editors March 23, 1992 and, in revised form, June 1, 1992.

1991 Mathematics Subject Classification. Primary 22D05, 22E05; Secondary 22C05.

Key words and phrases. Lie group, compact subgroup, inductive, maximal compact subgroup, locally compact group.

The final version of this article was written while the first author enjoyed again the hospitality of Tulane University. 
Theorem 1.1. In a Lie group $G$ with finitely many components, the partially ordered set $\mathscr{C}_{G}$ is inductive.

This means, we recall, that for any tower $\mathscr{T} \subseteq \mathscr{C}_{G}$ the subgroup $\overline{U \mathscr{T}}$ is compact.

Typical examples of Lie groups $G$ in which $\mathscr{C}_{G}$ fails to be inductive are the discrete groups

(i) $\Delta_{1}=\frac{1}{p^{\infty}} \mathbb{Z} / \mathbb{Z}$ where $\frac{1}{p^{\infty}} \mathbb{Z}=\left\{m / p^{n}: m, n \in \mathbb{Z}\right\}$.

(ii) $\Delta_{2}=(\mathbb{Z} / p \mathbb{Z})^{(\mathbb{N})}$, the countably infinite vector space over $\operatorname{GF}(p)$.

(iii) $\Delta_{3}=\bigoplus_{n \in \mathbb{N}} \mathbb{Z} / p_{n} \mathbb{Z}$ for a countable family of pairwise different primes $p_{n}$.

Using Theorem 1.1, the second author shows elsewhere [13] that for a Lie group $G$ the set $\mathscr{C}_{G}$ is inductive if and only if $G$ does not contain a discrete subgroup isomorphic to one of $\Delta_{1}, \Delta_{2}$, or $\Delta_{3}$. An illuminating example of a nondiscrete locally compact group $G$ for which $\mathscr{C}_{G}$ is not inductive is the additive group $\mathbb{Q}_{p}$ of $\mathrm{p}$-adic integers.

Actually, Theorem 1.1 is better than Theorem A. To see this let us consider the two properties of a topological group $G$ :

$\left(\mathbf{M}_{G}\right)$ Every element of $\mathscr{C}_{G}$ is contained in a maximal one.

$\left(\mathrm{I}_{G}\right) \mathscr{C}_{G}$ is inductive.

By Zorn's Lemma, $\left(\mathrm{I}_{G}\right)$ implies $\left(\mathrm{M}_{G}\right)$. If $H$ is any closed subgroup of $G$, then $\left(\mathbf{I}_{G}\right)$ implies $\left(\mathbf{I}_{H}\right)$. But $\left(\mathbf{M}_{G}\right)$ does not imply either $\left(\mathbf{I}_{G}\right)$ or $\left(\mathbf{M}_{H}\right)$. In $\S 4$ we shall prove

Example 1.2. There is a countable (discrete) group $G=\Delta_{4}$ with a subgroup $H$ such that the following conditions are satisfied:

(i) $H \cong \mathbb{Z}\left(2^{\infty}\right)$. In particular, $\left(\mathrm{I}_{G}\right)$ and $\left(\mathrm{M}_{H}\right)$ fail.

(ii) Every finite subgroup is cyclic and is contained in a maximal finite subgroup, i.e., $\left(\mathbf{M}_{G}\right)$ holds.

There is no a priori reason why a connected Lie group, say, could not contain a discrete subgroup isomorphic to $\Delta_{n}$. After all, $\operatorname{PSl}(2, \mathbb{R})$ contains the discrete subgroup $\operatorname{PSl}(2, \mathbb{Z})$, a complicated group which is isomorphic to the free product $\mathbb{Z}(2) * \mathbb{Z}(3)$ of cyclic groups of order 2 and 3 (see [12, p. 187]) whose commutator group is free [12, p. 201] and, thus, also contains a free group of countably many generators. But Theorem 1.1 prevents the occurrence of a discrete subgroup isomorphic to $\Delta_{n}$ for $n=1,2,3,4$ in a connected Lie group.

We shall provide in this note a proof of Theorem 1.1. This will also provide a new proof of Theorem A. Special cases of Theorem B shall come out in the wash. We shall not offer a new proof of Theorem B in its full generality nor of Theorem C. In fact, for a proof of a more comprehensive result in the direction of Theorem 1.1 we shall use Theorems A, B, and C. Nevertheless, one might compare our basic line of reasoning with the rather hard textbook passages of pp. 172-186 in Hochschild's book [7].

In any event, one needs a fixed point theorem of sorts, and we shall use one for a proof of Theorem 1.1. The difference is that here we use the idea of automorphism groups of convex cones in a vector space. Convex cones and compact groups go well together. (This was in fact used by the first author in a proof of the Theorem of Peter and Weyl [8].) 
Indeed, let $E$ denote a finite-dimensional real vector space and $C$ a closed convex pointed cone with $E=C-C$. A linear automorphism $g$ of $E$ with $g C=C$ is called an automorphism of $C$. If $v$ is any vector in the interior of $C$ and $G \subseteq$ Aut $E$ is any group of automorphisms then the orbit $G v$ is contained in the interior. If $G$ is compact then normalized Haar measure on $G$ allows us to find the barycenter $b=\int_{G} g v d g$ of $G v$ and its invariance shows that $G b=\{b\}$. Thus one has the elementary observation:

Lemma 1.3(a). Every compact group of automorphisms of a closed convex pointed cone in a finite-dimensional vector space has a fixed point in the interior of the cone.

However, the following converse holds, too (see [6]):

Lemma 1.3(b). If $v$ is a point in the interior of a closed convex pointed cone $C$ in a finite-dimensional vector space $E=C-C$, then the isotropy group (Aut $C)_{v}$ of all automorphisms of $C$ fuxing $v$ is compact.

Proof. The outline of a proof is readily understood: Let $E=C-C$ and let $C^{*}=\left\{\omega \in E^{*}:(\forall v \in V)\langle\omega, v\rangle \geq 0\right\}$ be the dual cone which has interior and is pointed. The group Aut $C$ acts by adjoint action on $E^{*}$ via $\langle g \cdot \omega, v\rangle=$ $\left\langle\omega, g^{-1} v\right\rangle$, and (Aut $\left.C\right)_{v}$ leaves the hyperplane $H=\left\{\omega \in E^{*}:\langle\omega, v\rangle=1\right\}$ invariant as a whole. Then it leaves the closed convex hull of $\left(H \cap C^{*}\right) \cup$ $-\left(H \cap C^{*}\right)$ invariant, which is-as is readily checked from the fact that $C^{*}$ has interior points and that $H \cap C^{*}$ is compact-a compact neighborhood of 0 in $E^{*}$. Thus (Aut $\left.C\right)_{v}$, a closed subgroup of Aut $E$, acts faithfully and equicontinuously on $E^{*}$ and is, therefore, compact.

Lemma 1.4. If $G$ denotes the automorphism group of a closed convex pointed cone $C$ in a finite-dimensional vector space $E=C-C$, then every maximal compact subgroup $K$ of $G$ is an isotropy group of $G$ at a vector $v$ in the interior of $C$.

Proof. If $K$ is a maximal compact subgroup of $G$, then it has a fixed point $v$ in the interior of $C$ by Lemma 1.3(a). The isotropy group $G_{v}$ is compact by Lemma 1.3(b), and it contains $K$. The maximality of $K$ then shows $K=$ $G_{v}$.

Notice that we are not claiming that every isotropy group $G_{v}$ is a maximal compact subgroup of $G$. In fact, only the next lemma, which is now easily proved, establishes, via the Lemma of Zorn, the existence of maximal compact subgroups. This is the main lemma which is at the core of our argument.

Lemma 1.5 (Main Lemma). Let $G$ denote a topological group acting linearly and continuously on a finite-dimensional real vector space $E$, leaving a closed convex pointed cone $C$ with $E=C-C$ invariant.

(i) If $\mathscr{D}$ denotes any upwards directed subset of $\mathscr{C}_{G}$ then there is a vector $v$ in the interior of $C$ such that the isotropy group $G_{v}$ at $v$ contains all members of $\mathscr{D}$.

(ii) If $G=$ Aut $C$ (acting by the natural action) then $\mathscr{C}_{G}$ is inductive.

Proof. (i) For each $K \in \mathscr{C}_{G}$ the set of fixed points under $K$, say $\operatorname{Fix}(K)$, is a vector subspace of $E$, meeting the interior of $C$ in $E$ by Lemma 1.3(a). If 
$K_{1} \subseteq K_{2}$ then $\operatorname{Fix}\left(K_{2}\right) \subseteq \operatorname{Fix}\left(K_{1}\right)$. If $\mathscr{D} \subseteq \mathscr{C}_{G}$ is an upwards directed set then $\operatorname{Fix}(\mathscr{D})=\{\operatorname{Fix}(K): K \in \mathscr{D}\}$ is a filterbasis of finite-dimensional nonzero vector spaces. For reasons of dimension every such filter basis contains a smallest element $\operatorname{Fix}(K), K \in \mathscr{D}$. Find $v \in \operatorname{Fix}(K)$ in the interior of $C$ in view of Lemma 1.3(a). Then, since $\operatorname{Fix}(K) \subseteq \operatorname{Fix}(H)$ for all $H \in \mathscr{D}$, the isotropy group $G_{v}$ of $v$ contains each $H \in \mathscr{D}$.

(ii) In this case $G_{v}$ is compact by Lemma 1.3(b) and, thus, is an upper bound of $\mathscr{D}$ in $\mathscr{C}_{G}$.

Definition 1.6. We shall call a topological group $G$ an ICG-group if the partially ordered set $\mathscr{C}_{G}$ of compact subsets is inductive.

The following remarks are rather obvious.

Lemma 1.7. (i) If $G$ is an ICG-group then every closed subgroup is an ICG-group.

(ii) If $N$ is a compact normal subgroup of $G$ then $G / N$ is an ICG-group if and only if $G$ itself is an ICG-group.

\section{LINEAR LIE GROUPS}

A first typical application turns to case of the vector space $\operatorname{Hom}(\mathscr{H}, \mathscr{H})$ of all endomorphisms of the real or complex $n$-dimensional Hilbert space. We organize our thoughts by generalizing slightly.

Recall that a real or complex $C^{*}$-algebra is an involutive Banach algebra satisfying $\left\|x^{*} x\right\|=\|x\|^{2}$ for all $x$. The set $\operatorname{Hom}(\mathscr{H}, \mathscr{H})$ is a prototypical $C^{*}$-algebra.

Let $A$ denote a finite-dimensional real or complex $C^{*}$-algebra with identity and $G$ its group of units. The group $U=\left\{g \in G: g^{-1}=g^{*}\right\}$ of unitary elements is contained in the unit ball of the finite-dimensional real vector space $\operatorname{Hom}(A, A)$ since for a unitary element $g$ we have $\|g\|^{2}=\left\|g^{*} g\right\|=1$. Since $U$ is defined by the equation $g^{*} g=1$, it is closed in $\operatorname{Hom}(A, A)$ and, thus, is a compact subgroup of $G$. The set $A_{h}=\left\{a \in A: a^{*}=a\right\}$ of all hermitean elements is a vector subspace of $A$, and the group $G$ acts on it linearly via $g \cdot a=g a g^{*}$.

An element $x \in A$ is called positive if it can be written $x=a^{*} a$ (which is tantamount to saying that its spectrum is contained in the nonnegative real axis). The set of all positive elements is a closed convex pointed cone $C$ in $A_{h}$ such that $A_{h}=C-C$ and that $G \cdot C=C$.

Given that every positive element $x$ in a $C^{*}$-algebra has a positive square root $y$, and given the viewpoint of $\S 1$, the following is now elementary:

Lemma 2.1. (i) If $x$ is any positive element and $y$ a positive square root, then $y^{-1} G_{x} y \subseteq U$. In particular, $G_{x}$ is compact.

(ii) Every compact subgroup of $G$ is conjugate to a subgroup of $U$.

(iii) $U$ is a maximal compact subgroup of $G$ and all maximal subgroups of $G$ are conjugate.

Proof. (i) An element $k$ is in $G_{x}$ iff $k x k^{*}=k \cdot x=x$. This is equivalent to $x^{-1} k^{-1} x=k^{*}$. In view of $x=y^{2}$ this means $y^{2} k^{*} y^{2}=k^{-1}$, and because of $y=y^{*}$ this implies

$$
\left(y^{-1} k y\right)^{-1}=y^{-1} k^{-1} y=y k^{*} y^{-1}=\left(\left(y^{-1}\right)^{*} k y^{*}\right)^{*}=\left(y^{-1} k y\right)^{*} .
$$

Thus $y^{-1} k y \in U$. 
(ii) Suppose that $K$ is a compact subgroup of $G$. By Lemma 1.2 there is a vector $v \in \operatorname{int} C$ fixed by $K$. By (i) there is a $w \in C$ such that $w^{-1} G_{v} w \subseteq U$. Hence $w^{-1} K w \subseteq w^{-1} G_{v} w \subseteq U$.

(iii) If $U \subseteq K$ with $K$ as in the proof of (ii), then $U \subseteq K \subseteq w U w^{-1}$. Since $U$ is a Lie group, equality follows and we have $U=K$. Thus $U$ is maximal compact. The remainder then follows from (ii).

Proposition 2.2. Let $A$ denote a finite-dimensional real or complex $C^{*}$-algebra with identity and $G$ its group of units. Then $G$ is an ICG-group.

Proof. Every tower $\mathscr{D}$ in $\mathscr{C}_{G}$ is bounded by an isotropy group $G_{v}$ for a $v \in$ int $C$ by Lemma $1.5(\mathrm{i})$. But $G_{v}$ is compact by Lemma $1.8(\mathrm{i})$.

A topological group $G$ is called a linear Lie group if it is isomorphic (algebraically and topologically) to a closed subgroup of some group $\mathrm{Gl}(n, \mathbb{R})$ or $\mathrm{Gl}(n, \mathbb{C})$.

Corollary 2.3. Every linear Lie group is an ICG-group.

Proof. By Lemma 1.7(i) it suffices to note that all full real or complex linear groups are ICG-groups. But that follows at once from Proposition 1.9 with the $C^{*}$-algebra $A$ of all $n \times n$ real or complex matrices.

As mentioned above, not all Lie groups are ICG-groups, but we shall show that all those with finitely many components are.

We note that the basic facts on $C^{*}$-algebras used above are elementary linear algebra in the case of full matrix algebras.

\section{LIE GROUPS AND LOCALLY COMPACT GROUPS}

In this section we return to some traditional methods in the structure theory of locally compact groups and, combining these with the results obtained so far, arrive at the main results.

Lemma 3.1. Let $G$ be a topological group containing a normal, open subgroup $N$ such that $N$ and $G / N$ are ICG-groups. Then $G$ is an ICG-group.

Proof. Since $N$ is open, it is also closed. Moreover, $G / N$ is a discrete group. Let $\pi: G \rightarrow G / N$ denote the quotient map of $G$ onto $G / N$, and let $\mathscr{D}$ be a tower of compact subgroups of $G$. Since $N$ is an ICG-group, $\bigcup_{K \in \mathscr{D}} K \cap N$ is compact. Now, $N$ is open and closed. Hence, setting $C=\bar{U} \mathscr{D}$, we have

$$
\overline{\bigcup_{K \in \mathscr{D}} K \cap N}=\overline{(\bigcup \mathscr{D}) \cap N}=C \cap N .
$$

This shows that $C \cap N$ is a compact, open, and normal subgroup of $C$. Now, $G / N$ is an ICG-group. Thus $\overline{\bigcup\{\pi(K): K \in \mathscr{D}\}}=\overline{\pi(\bigcup \mathscr{D})}$ is compact. Since $G / N$ is discrete, this set is finite and equals $\pi(C)$. In view of $C /(C \cap N) \cong$ $C N / N=\pi(C)$, we conclude that $C /(C \cap N)$ is finite, too. Since we have already seen that $C \cap N$ is compact, we finally infer that $C$ is compact.

Note that the converse fails: Take the discrete groups $G=\mathbb{Q}, N=\mathbb{Z}$. 
We will now take a closer look at Z-groups, i.e., locally compact groups which are compact modulo their center. The following result, which is due to Grosser and Moskowitz, is crucial for the study of Z-groups. For a proof see [5, Corollary 2 of Theorem 4.4].

Lemma 3.2. Let $G$ be a locally compact group, and let $Z$ denote the center of $G$. Assume that $G / Z$ is compact. Then there exist closed normal subgroups $V \cong \mathbb{R}^{n}$ and $H$ and $N$ of $G$ such that $G \cong V \times H, N$ is compact and open in $H$, and $H / N$ is abelian.

Lemma 3.3. Let $G$ be a Z-group, and let $Z$ denote the center of $G$. Then $G$ is an ICG-group if and only if $Z$ is an ICG-group.

Proof. If $G$ is an ICG-group then $Z$ is also an ICG-group (Lemma 1.7.i). Conversely, assume that $Z$ is an ICG-group. By Lemma 3.2, there exist closed normal subgroups $V, N$, and $H$ as in Lemma 3.2. Since every compact subgroup of $G$ is contained in $H$, it follows that $G$ is an ICG-group if and only if $H$ is an ICG-group. We have $Z \cong V \times Z(H)$, whence $Z(H)$ is an ICG-group since $Z$ is an ICG-group. Moreover, $G / Z \cong H / Z(H)$, and thus $H / Z(H)$ is compact. We have $Z(H) /(N \cap Z(H)) \cong(N \cdot Z(H)) / N$. Since $Z(H)$ is an ICG-group and $N \cap Z(H)$ is compact, $Z(H) /(N \cap Z(H))$ and thus $(N \cdot Z(H)) / N$ is an ICG-group by Lemma 1.7 (ii). Hence, $N \cdot Z(H)$ is an ICG-group by Lemma 1.7(ii) again. Now $H /(N \cdot Z(H))$ is compact, and $N \cdot Z(H)$ is an open normal subgroup of $H$. Then $H$ is an ICG-group by Lemma 3.1 .

Lemma 3.4. Let $G$ be a topological group containing a closed, normal subgroup $N$ such that $G / N$ is an ICG-group. Assume that every closed subgroup $H$ of $G$ which contains $N$ and has compact factor group $H / N$ is an ICG-group. Then $G$ is an ICG-group.

Proof. Let $\pi: G \rightarrow G / N$ denote the quotient map, and let $\mathscr{D}$ be a tower of compact subgroups of $G$. Then $\pi(\mathscr{D})$ is a tower of compact subgroups of $G / N$. Since $G / N$ is an ICG-group, there exists a compact subgroup $K$ of $G / N$ such that $\bigcup \pi(\mathscr{D}) \subseteq K$. Now $\pi^{-1}(K) / N$ is isomorphic to $K$ and, thus, is compact. By hypothesis, $\pi^{-1}(K)$ is an ICG-group. Since $\mathscr{D}$ is a tower in $\pi^{-1}(K)$, we conclude that $\bigcup \mathscr{D}$ has compact closure in $\pi^{-1}(K)$ and, therefore, in $G$.

Proposition 3.5. Let $G$ be a locally compact group, and let $Z$ denote the center of $G$. If $Z$ and $G / Z$ are ICG-groups then $G$ is an ICG-group.

Proof. This is immediate from Lemmas 3.3 and 3.4.

Lemma 3.6. Every connected semisimple Lie group $G$ is an ICG-group.

Proof. Let $\mathfrak{g}$ denote the Lie algebra of $G$ and Ad: $G \rightarrow \operatorname{Aut}(\mathfrak{g})$ the adjoint representation of $G$. Then $\operatorname{Aut}(\mathfrak{g})$ is a closed subgroup of $\mathrm{Gl}(\mathfrak{g})$ whose identity component is $\operatorname{Ad}(G)$, since $G$ is semisimple. Hence, $\operatorname{Ad}(G)$ is a linear Lie group and, therefore, an ICG-group by Corollary 2.3. The kernel of Ad is the center $Z$ of $G$. Hence, $G / Z \cong \operatorname{Ad}(G)$ is an ICG-group. But $Z$ is a discrete finitely generated abelian group and, thus, is clearly an ICG-group. Hence, by Proposition 3.5, $G$ is an ICG group. 
We now deal with the radical.

Lemma 3.7. Suppose that $N$ is a normal subgroup of a locally compact group $G$ such that $G / N$ is compact and $N$ is a vector group. Then $G$ is an ICG-group. Proof. We know (e.g., from [3, Chapter VII, §3, no. 2, Proposition 3, p. 30], or from [9, p. 30]) that $G$ is isomorphic to a semidirect product $\mathbb{R}^{n} \rtimes K$ with a compact group $K$. Since $G$ has a compact normal subgroup modulo which it is a Lie group, it is no loss of gerierality to assume that $G$, hence $K$, is a Lie group. The kernel of the action of $K$ on $\mathbb{R}^{n}$ is normal in $G$ and compact; we may again assume that it is trivial. Then we may identify $K$ with a subgroup of $\mathrm{O}(n)$, and by Lemma 1.7(i) we may assume that $G=\mathbb{R}^{n} \rtimes \mathrm{O}(n)$. However, this group is isomorphic to a closed subgroup of $\mathrm{Gl}(n+1, \mathbb{R})$; for we have

$$
G \cong\left\{\left(\begin{array}{cc}
A & v \\
0 & 1
\end{array}\right) \mid A \in \mathrm{O}(n) \text { and } v \in \mathbb{R}^{n}\right\},
$$

where we identified $\mathbb{R}^{n}$ with the vector space of $(n \times 1)$-matrices. Thus Corollary 2.3 implies the assertion.

Now we are ready to deal with connected Lie groups.

Theorem 3.8. Every connected Lie group is an ICG-group.

Proof. We consider the radical $R$ of $G$ and prove the assertion by induction with respect to the dimension of $R$. If $\operatorname{dim} R=0$, that is, $R=\{1\}$, then the assertion is true by Lemma 3.6. Now suppose that the assertion has been proved for radicals with dimension $<n$ and that $\operatorname{dim} R=n$. If $\mathfrak{v}$ is the last term of the commutator series of $\mathfrak{r}$, then $\mathfrak{v}$ is a characteristic ideal of $\mathfrak{r}$ and thus is an abelian ideal of $\mathfrak{g}$. Then $\overline{\exp \mathfrak{v}}$ is a closed connected normal abelian subgroup. Let $A$ denote a connected closed nonzero abelian normal subgroup $A$ of $G$ of minimal positive dimension. By induction hypothesis, $G / A$ is an ICG-group. If $A$ contains a nonzero torus subgroup, then, since this subgroup is characteristic in $A$, by the minimality of $A$, the group $A$ itself is a torus and is therefore central in $G$. In this case $G$ is an ICG-group by Lemma 1.7(ii). Now assume that $A$ has no torus subgroup. Then $A$ is a vector group. Let $\mathscr{D}$ be an up-directed family of compact subgroups. Now $\overline{\bigcup_{C \in \mathscr{D}} C A / A}$ is a compact group $K / A$. Then $K$ is a subgroup of the type discussed in the preceding Lemma 3.7 and, thus, is an ICG-group. Hence, the union of $\mathscr{D}$ is contained in a compact group, and thus $G$ is an ICG-group. The induction is complete.

Corollary 3.9. If $G$ is a locally compact group such that $G / G_{0}$ is compact then $G$ is an ICG-gorup.

Proof. Since $G$ contains a compact normal subgroup modulo which it is a Lie group, in view of Lemma 1.7 (ii) we may assume that $G$ is a Lie group. Now $G_{0}$ is an ICG-group by the preceding proposition. Now Lemma 3.1 proves the rest.

In particular, Corollary 3.9 proves Theorem 1.1 of the introductory section.

For our final result we shall use nontrivial facts in the following lemma. The background for these results is dispersed in the literature. For Theorems B 
and $\mathrm{C}$ we refer to Hochschild's book [7, 180ff]. The referee points out that a forerunner is contained in [10]. After the paper was written it came to our attention that Bagley, $\mathrm{Wu}$, and Yang had independently proved part of a slightly more general version of part (iv) in [1, Lemma 1.8].

Lemma 3.10. Suppose that $G$ is a locally compact group such that $G / G_{0}$ is compact. Then the following conclusions hold:

(i) Every compact subgroup of $G$ is contained in a maximal compact subgroup $K$, and every such satisfies $G=G_{0} K=K G_{0}$.

(ii) If $K$ is maximal compact in $G$ then $K_{0}=K \cap G_{0}$ and this group is maximal compact in $G_{0}$.

(iii) If $K$ is a compact subgroup satisfying $G=G_{0} K$ such that $K_{0}$ is maximal compact in $G_{0}$ then $K$ is maximal compact in $G$.

(iv) If $H$ is a closed subgroup of $G$ containing $G_{0}$ and $K$ is maximal compact in $G$ then $H \cap K$ is maximal compact in $H$.

Proof. The group $G$ contains a compact subgroup $N$ such that $G / N$ is a Lie group. Theorems A, B, and C generalize quickly to our present case. We shall notably use Theorem $\mathrm{C}$ and conclude that for a maximal compact subgroup $K$ of $G$ there is a subspace $E \subseteq G$ homeomorphic to $\mathbb{R}^{n}$ such that $(k, e) \mapsto$ $k e: K \times E \rightarrow G$ is a homeomorphism. Every element $g \in G$, therefore, has a unique decomposition $g=k_{g} e_{g}$ with $k_{g} \in K$ and $e_{g} \in E$.

(i) is a ready consequence of these observations.

(ii) Clearly, by the preceding remarks, $G_{0}=K_{0} E$. If $k \in K \cap G_{0}$ then $k=k_{0} e$ for $k_{0} \in K_{0}$ and $e \in E$, and thus $1=\left(k^{-1} k_{0}\right) e$ proving $k^{-1} k_{0}=1$, i.e., $k \in K_{0}$. Since trivially $K_{0} \subseteq K \cap G_{0}$, we have $K_{0}=K \cap G_{0}$.

Now $G_{0} / K_{0} \sim E$ is homeomorphic to a euclidean space. We shall show that $K_{0}$ is maximal compact in $G_{0}$ : Let $C$ denote a maximal compact subgroup of $G_{0}$ containing $K_{0}$; then $G_{0}=C E$ (topologically direct), and thus $G_{0} / K_{0}$ is homeomorphic to $C / K_{0} \times E$ which is homeomorphic to a euclidean space only if $C=K_{0}$.

(iii) Suppose that $K \subseteq C \subseteq G$ with a compact group $C$. Then $C=$ $K(C \cap E)$. Thus $c \in C$ can be written $c=k e$ with $e \in G_{0}$. Now $e=$ $k^{-1} c \in C \cap G_{0}$. But $K_{0} \subseteq C \cap G_{0}$ and $C \cap G_{0}$ is compact. But $K_{0}$ is maximal compact by hypothesis. Hence, $C \cap G_{0}=K_{0}$. Thus $e \in K_{0} \subseteq K$, and therefore $c=k e \in K$. We have shown that $K$ is maximal compact in $G$.

(iv) Since $G_{0} \subseteq H$, we have $H=(K \cap H) E$. (Indeed the right side is in the left, and if $h=k_{h} e_{h}$ is in $H$ then $k_{h}=h e_{h}^{-1} \in H G_{0}=H$, i.e., $k_{h} \in K \cap H$.) Thus $H=(K \cap H) H_{0}$ and $K_{0}=K \cap H_{0}$ is maximal compact in $H_{0}$ by (ii). Hence $K \cap H$ is maximal compact in $H$ by (iii).

Our conclusive result, reducing the classification of locally compact ICGgroups to that of zero-dimensional locally compact ICG-groups, is the following:

Theorem 3.11. For a locally compact group $G$ the following conditions are equivalent :

(1) $G / G_{0}$ is an ICG-group.

(2) $G$ is an ICG-group.

Proof. (1) implies (2). Suppose that $\mathscr{D}$ is a directed set of compact subgroups. Then $\overline{\bigcup_{D \in \mathscr{D}} D G_{0} / G_{0}}$ is a compact group, say $H / G_{0}$, since $G / G_{0}$ is an 
ICG-group. Then $H$ is an ICG-group by Corollary 3.9. It follows that $\cup \mathscr{D}$ is contained in a compact group. Hence, $G$ is an ICG-group.

(2) implies (1). Let $\mathscr{D}$ be a tower of compact subgroups of $G / G_{0}$, and let $\pi: G \rightarrow G / G_{0}$ denote the quotient map. We want to show that the closure of $\bigcup \mathscr{D}$ is compact.

Now, $\pi^{-1}(K) / G_{0}$ is compact for every $K \in \mathscr{D}$. Hence, by Lemma $3.10(\mathrm{i})$, there exists a maximal compact subgroup $M$ of $\pi^{-1}(K)$.

If we set $M_{C}:=\pi^{-1}(C) \cap M$ for $C \in \mathscr{D}$ with $C \subseteq K$ then

(*) $\quad M_{C}$ is maximal compact in $\pi^{-1}(C)$ by Lemma 3.10(iv) and the function $C \mapsto M_{C}$ is order preserving.

It is our next aim to construct an order-preserving function $K \mapsto M_{K}: \mathscr{D} \rightarrow$ $\mathscr{C}_{G}$ such that $M_{K}$ is maximal compact in $\pi^{-1}(K)$ for every $K \in \mathscr{D}$. We will do this with the help of Zorn's Lemma.

Let $\mathbf{J}$ denote the set of all order-preserving functions $K \mapsto M_{K}: \mathscr{D}^{\prime} \rightarrow \mathscr{C}_{G}$ for some initial section $\mathscr{D}^{\prime}$ of $\mathscr{D}$ such that $M_{K}$ is maximal compact in $\pi^{-1}(K)$ for all $K \in \mathscr{D}^{\prime}$. Remark (*) shows that $\mathbf{J}$ is not empty. The set $\mathbf{J}$ is partially ordered by restriction, i.e., a function $\phi$ precedes a function $\psi$ if it is a restriction of $\psi$. With this order, $\mathbf{J}$ is inductive and, thus, contains a maximal element, say $C^{\prime} \mapsto C_{K}^{\prime}: \mathscr{D}^{\prime} \rightarrow \mathscr{C}_{G}$. We claim that $\mathscr{D}^{\prime}=\mathscr{D}$. By way of contradiction assume that $\mathscr{D}^{\prime} \neq \mathscr{D}$. There is at least one element $L \in \mathscr{D} \backslash \mathscr{D}^{\prime}$. Since $\mathscr{D}^{\prime}$ is an initial section, we have $C^{\prime} \subseteq L$ for all $C^{\prime} \in \mathscr{D}^{\prime}$ and, thus,

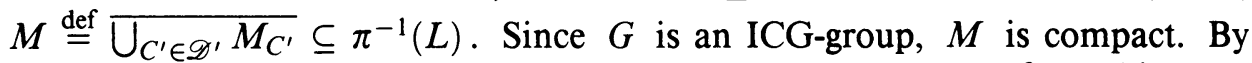
Lemma 3.10(i), there exists a maximal compact subgroup $M_{L}$ of $L$ which satisfies both $\pi\left(M_{L}\right)=L$ and $M \subseteq M_{L}$. Set $\mathscr{D}^{\prime \prime}=\{C \in \mathscr{D}: C \subseteq L\}$ and $\widetilde{M}_{C}=\pi^{-1}(C) \cap M_{L}$ for all $C \in \mathscr{D}^{\prime \prime}$. By Remark (*) the group $\widetilde{M}_{C}$ is maximal compact in $\pi^{-1}(C)$ for all $C \in \mathscr{D}^{\prime \prime}$, and $C \mapsto \widetilde{M}_{C}$ is order preserving. Moreover, for all $C^{\prime} \in \mathscr{D}^{\prime}$ we have $M_{C^{\prime}} \subseteq M \subseteq M_{L}$ and, thus, $M_{C^{\prime}} \subseteq \widetilde{M}_{C}^{\prime}$. The maximality of $M_{C^{\prime}}$ then shows equality. Hence, $C \mapsto \widetilde{M}_{C}: \mathscr{D}^{\prime \prime} \rightarrow \mathscr{C}_{G}$ is a proper extension of $C^{\prime} \mapsto M_{C^{\prime}}: \mathscr{D}^{\prime} \rightarrow \mathscr{C}_{G}$. This is a contradiction to the maximality of the latter function.

Thus, there exists an order-preserving function $K \mapsto M_{K}: \mathscr{D} \rightarrow \mathscr{C}_{G}$ which assigns to each $K \in \mathscr{D}$ a maximal compact subgroup $M_{K}$ of $\pi^{-1}(K)$. Since $G$ is an ICG-group, $\bigcup_{K \in \mathscr{D}} M_{K}$ has compact closure $M$ in $G$. Now, $\pi(M)$ is compact, and $\cup \mathscr{D}$ is contained in $\pi(M)$ since $\pi\left(M_{K}\right)=K$ for $K \in \mathscr{D}$ by Lemma $3.10(\mathrm{i})$. This proves that $\mathscr{C}_{G / G_{0}}$ is inductive.

\section{AN EXAMPLE}

We set $\mathbb{N}_{0}=\{0,1,2, \ldots\}$ and let $X$ denote an alphabet $\left\{x_{1}, x_{2}, \ldots\right\}$ of distinct letters. Now we define $G$ to be the group

$$
\left\langle x_{1}, \ldots ; x_{1}^{4}=1, x_{n+1}^{4}=x_{n}^{2}, n=1,2, \ldots\right\rangle
$$

generated by the $x_{j}$ subject to the relations stated. We shall also use an additional letter $x_{0}$ by setting $x_{0}=x_{1}^{2}$. We let $H$ denote the subgroup generated by all $x_{n}^{2}, n=1,2, \ldots$. Writing temporarily $y_{n}=x_{n}^{2}$ we obtain different generators of $H$ subject to the relations $y_{1}^{2}=1$ and $y_{n+1}^{2}=y_{n}$. This remark shows that $H$ is isomorphic to $\mathbb{Z}\left(2^{\infty}\right)$. The partially ordered set $\mathscr{C}_{G}$ of finite 
subgroups of $G$, due to the presence of $H$, is not inductive, and in $H$ there are no maximal elements of $\mathscr{C}_{G}$ whatsoever. However, we shall prove the following proposition.

Proposition 4.1. (i) Every finite subgroup of $G$ is conjugate to a subgroup of some $\left\langle x_{n}\right\rangle, n \in \mathbb{N}$.

(ii) Each $\left\langle x_{n}\right\rangle$ and every one of its conjugates is maximal in $\mathscr{C}_{G}$.

In particular, every element of $\mathscr{C}_{G}$ is contained in a maximal element of $\mathscr{C}_{G}$. The group $G$, therefore, is an example of a group labelled $\Delta_{4}$ in Example 1.2.

We shall now prove Proposition 4.1 in several steps.

Proof. We set $S=H \cup \dot{\cup}$.

(a) Since $x_{n}^{2 m} \in H$ and $x_{n}^{2 m+1}=x_{n} u=u x_{n}$ for all $n \in \mathbb{N}_{0}, m \in \mathbb{Z}$ and some $u \in H$, every element $g \in G$ can be written in the form

$$
g=u_{1} \cdots u_{n}, \quad u_{j} \in S, \quad j=1, \ldots, n .
$$

If $x=\epsilon X$ and $h \in H$ then $x h=h x$ if and only if $h \in\langle x\rangle$

Let $\# M$ denote the number of elements in a finite set $M$. We shall associate with each $1 \neq g \in G$ two natural numbers:

$$
\begin{aligned}
& n(g)=\min \{n \in \mathbb{N}:\left.\left(\exists u_{1}, \ldots, u_{n} \in S\right) g=u_{1} \cdots u_{n}\right\}, \\
& \ell(g)=\min \{\ell \in \mathbb{N}:\left(\exists u_{1}, \ldots, u_{n} \in S\right) g=u_{1} \cdots u_{n}, \\
&\text { and } \left.\ell=\#\left\{j=1, \ldots, n: u_{j} \in X\right\}\right\} .
\end{aligned}
$$

We note that $\ell(g) \leq n(g) \leq 2 \ell(g)+1$ and write $n(1)=\ell(1)=0$.

(b) We shall call an element $g \in G$ reduced if it cannot be written in the form $g=a g^{\prime} a^{-1}$ with $a, g^{\prime} \in G$ and $n\left(g^{\prime}\right)<n(g)$. Every element is the conjugate of a reduced element. We will deal mostly with reduced elements.

(c) Claim: If $g=u_{1} \cdots u_{n(g)}$ is reduced then the configuration

$$
\text { " } \ell(g) \geq 1, u_{1} \in H, \text { and } u_{n(g)} \in H "
$$

is not possible. Proof: Assume we had this configuration. Set $a=u_{n(g)}^{-1}$, $v_{1}=u_{n(g)} u_{1} \in H \subseteq S$, and $v_{j}=u_{j} \in S$ for $j=2, \ldots, n(g)-1$. Note $g=a\left(v_{1} \cdots v_{n(g)-1}\right) a^{-1}$. Then $g$ would not be reduced. Contradiction.

(d) Claim: If $g=u_{1} \cdots u_{n(g)}$ is reduced, $\ell(g) \geq 2, u_{1} \in H, u_{n(g)} \in X$ then $u_{2} \in X$, and if also $u_{1} \in\left\langle u_{n(g)}\right\rangle$ then $u_{2} \neq u_{n(g)}$. (A dual assertion holds for $u_{1} \in X$ and $u_{n(g)} \in H$.) Proof: If $u_{1} \in H$ then $u_{2} \neq H$ by the definition of $n(g)$. Assume $u_{2}=u_{n(g)} \in X$ and $u_{1} \in\left\langle u_{2}\right\rangle=\left\langle u_{n(g)}\right\rangle$. Then set $a=u_{n(g)}^{-1}$, $v_{1}=u_{1} u_{2}^{2} \in H \subseteq S, v_{j}=u_{j+1} \in S$ for $j=2, \ldots, n(g)-2$, and note

$$
a\left(v_{1} \cdots v_{n(g)-2}\right) a^{-1}=u_{n(g)}^{-1} u_{2} u_{1} u_{3} \cdots u_{n(g)-1} u_{n(g)}=g \text {. }
$$

Thus $g$ would not be reduced. Contradiction.

(e) Claim: If $g=u_{1} \cdots u_{n(g)}$ is reduced, $\ell(g) \geq 2, u_{1}, u_{n(g)} \in X$ then $u_{1} \neq u_{n(g)}$. Proof: Assume that $u_{1}=u_{n(g)} \stackrel{\text { def }}{=} a \in X$. Set $v_{j}=u_{j+1} \in S$ for $j=1, \ldots, n(g)-2, v_{n(g)-1}=u_{n(g)}^{2} \in H \subseteq S$. Then $g=a v_{1} \cdots v_{n(g)-1} a^{-1}$ and this element would not be reduced. Contradiction.

(f) Claim: If $g=u_{1} \cdots u_{n(g)}$ is reduced and the order $o(g)$ of $g$ is finite, then $g \in\left\langle x_{n}\right\rangle$ for some $n \in N$. Proof: The assertion is clear for $g \in H$. Thus suppose $\ell(g) \geq 1$. By (c) we have the following cases: 
Case 1. $u_{1} \in H, u_{n(g)} \in X$.

Case 2. $u_{1} \in X, u_{n(g)} \in H$.

Case 3. $u_{1}, u_{n(g)} \in X$.

Case 2 is analogous to Case 1 . In Case 1 there are subcases:

Case $1(\mathrm{a}) . u_{1} \notin\left\langle u_{n(g)}\right\rangle$. Then for all natural numbers $p$ we have

$$
g^{p}=u_{1} \cdots u_{n(g)} u_{1} \cdots u_{n(g)} u_{1} \cdots \quad \cdots u_{n(g)}
$$

and $\ell\left(g^{p}\right)=p \ell(g)$, since $u_{1}$ and $u_{n(g)}$ do not commute and no reduction of the overall number of letters from $X$ is possible. This event is ruled out by $o(g)<\infty$.

Case $1(\mathrm{~b}) . \quad u_{1} \in\left\langle u_{n(g)}\right\rangle$. We now assume $\ell(g) \geq 2$. Then by (d) we have $u_{2}=x \neq y=u_{n(g)}$ with $x, y \in X$. Now

$$
\begin{aligned}
g^{p} & =u_{1} \cdots u_{n(g)} u_{1} \cdots u_{n(g)} u_{1} \cdots \quad \cdots u_{n(g)} \\
& =u_{1} \cdots u_{n(g)-1} u_{1} y x u_{3} \cdots u_{n(g)-1} u_{1} y x u_{3} \cdots \quad \cdots y,
\end{aligned}
$$

whence $\ell\left(g^{p}\right)=p \ell(g)$ since $x$ and $y$ do not commute and no reduction of the overall number of letters from $X$ is possible. This is inconsistent with $o(g)<\infty$.

Case 3. By Claim (e) we know $u_{1} \neq u_{n(g)}$, whence $\ell\left(g^{p}\right)=p \ell(g)$ follows as in the previous cases. Thus we are left with Case $1(\mathrm{~b})$ and $\ell(g)=1$. This means $g=h x_{n}$ with $h \in\left\langle x_{n}\right\rangle$ and, thus, $g \in\left\langle x_{n}\right\rangle$. This completes the proof of Claim (f).

(g) Claim: The only element of order 2 in $G$ is $x_{0}=x_{1}^{2}$, and this element is central. Proof: Since $x_{0}$ commutes with all $x_{n}$, it is central in $G$. Let $1 \neq g \in$ $F, g^{2}=1$. By (f) there is an $n \in \mathbb{N}$ and $a \in G$ such that $a g a^{-1} \in\left\langle x_{n}\right\rangle$. The only element of order 2 in $\left\langle x_{n}\right\rangle$ is $x_{0}$. Hence, $a g a^{-1}=x_{0}$, i.e., $g=a^{-1} x_{0} a$. Thus $g=x_{0}$.

(h) Claim: Let $E$ be a finite subgroup of $G$. Then $E$ is cyclic. Proof: By induction on $|E|$. So assume that $E$ is a noncyclic subgroup of minimal order. By (f) the group $E$ is a 2-group, and by (g) and our assumption it properly contains $\left\{1, x_{0}\right\}$. Define a surjective morphism $\pi: G \rightarrow\langle z\rangle * G$ onto the free product of a group of order 2 and $G$ by setting $\pi\left(x_{0}\right)=1, \pi\left(x_{1}\right)=z$, and $\pi\left(x_{n+1}\right)=x_{n}$ for $n=1,2, \ldots$. We observe that $\pi$ is well defined and has $\left\{1, x_{0}\right\}$ as kernel. Since $x_{0} \in E$, we record that $|\pi(E)|=|E| / 2<|E|$. As a finite subgroup of $\langle z\rangle * G$ the group $\pi(E)$ is contained either in a conjugate of $\langle z\rangle$ or in a conjugate of $G$. In the first case, $\pi(E)$ has order 2 and so $E$ has order 4 and is cyclic by $(\mathrm{g})$. This contradicts our assumption that $E$ is not cyclic. Thus w.l.g. we assume $\pi(E) \subseteq G$. Because of $|\pi(E)|<|E|$ and the minimality assumption on the order of $E$, we conclude that $\pi(E)$ is cyclic. Thus $E=\left\langle g, x_{0}\right\rangle$ for some $g \in G$. By (f) the group $\langle g\rangle$ is a cyclic 2-group. Then by $(\mathrm{g})$ some power of $g$ equals $x_{0}$. Hence $E=\langle g\rangle$, a contradiction to our assumption on $E$. This proves Claim (h).

With the proof of this claim we are done with part (i) of Proposition 4.1. The next claim will complete its proof.

(i) Claim: The groups $\left\langle x_{n}\right\rangle$ are maximally finite. Proof: Suppose that $\left\langle x_{n}\right\rangle \subseteq$ $F$ where $F$ is a finite subgroup of $G$. By (h) there is an $a \in G$ and a $p \in \mathbb{N}$ such that $F \subseteq\left\langle a x_{p} a^{-1}\right\rangle$, and for order reasons we have $n \leq p$. We must show $n=p$, because then for order reasons $\left\langle x_{n}\right\rangle \subseteq F \subseteq\left\langle a x_{p} a^{-1}\right\rangle$. Thus assume 
$n<p$. Then $a^{-1} x_{n} a$ is contained in a proper subgroup of $\left\langle x_{p}\right\rangle$. The largest proper subgroup is generated by $x_{p}^{2}$. But then $a^{-1} x_{n} a \in\left\langle x_{n}^{2}\right\rangle \subseteq H$. Now let $P=*_{m=1}^{\infty}\left\langle z_{m}\right\rangle$ denote the free product of cyclic groups of order 2 . There is a surjective homomorphism $\phi: G \rightarrow P$ defined by $\phi\left(x_{m}^{2}\right)=1$ for $m \in \mathbb{N}_{0}$ and $\phi\left(x_{m}\right)=z_{m}$ for $m \in \mathbb{N}$. Now $z_{n}=\phi\left(x_{n}\right) \in \phi\left(a H a^{-1}\right)=\phi(a) \phi(H) \phi(a)^{-1}=$ $\phi(a)\{1\} \phi(a)^{-1}=\{1\}$, and this is a contradiction.

\section{REFERENCES}

1. R. W. Bagley, T. S. Wu, and J. S. Yang, Inverse limits and dense immersions of locally compact groups, Bull. Inst. Math. Acad. Sinica 19 (1991), 97-124.

2. N. Bourbaki, Groupes et algèbres de Lie, Chapitres 1-9, Hermann (1-8), Masson (9), Paris, 1964-1982.

3. —_ Intégration, Chapters 7 et 8, Hermann, Paris, 1963.

4. V. M. Glushkov, Structure of locally bicompact groups and Hilbert's fifth problem, Uspekhi Mat. Nauk (N.S.) 12 (1957), no. 2(74), 3-41; English transl., Amer. Math. Soc. Transl. Ser. 215 (1960), 55-93.

5. S. Grosser and M. Moskowitz, On central topologcial groups, Trans. Amer. Math. Soc. 27 (1967), 317-340.

6. J. Hilgert, K. H. Hofmann, and J. D. Lawson, Lie groups, convex cones, and semigroups, Oxford Univ. Press, London, 1989.

7. G. Hochschild, Theory of Lie groups, Holden-Day, San Francisco, 1965.

8. K. H. Hofmann, Finite dimensional submodules of G-modules for a compact group, Proc. Cambridge Philos. Soc. 69 (1969), 47-52.

9. K. H. Hofmann and P. S. Mostert, Splitting in topological groups, Mem. Amer. Math. Soc., No. 63, Amer. Math. Soc., Providence, RI, 1963.

10. D. Montgomery, Simply connected homogeneous spaces, Proc. Amer. Math. Soc. 1 (1950), 467-469.

11. D. Montgomery and L. Zippin, Topological transformation groups, Interscience, New York, 1955.

12. W. Specht, Gruppentheorie, Springer-Verlag, Berlin, 1956.

13. C. Terp, Lie groups whose set of compact subgroups is inductive, Dissertation, Technische Hochschule, Darmstadt, 1991.

14. _ On locally compact groups whose set of compact subgroups is inductive, Sem. Sophus Lie 1 (1991), 73-80.

Fachbereich Mathematik, Technische Hochschule Darmstadt, Schlossgartenstr. 7, D-64380 DARMSTADT, GERMANY

E-mail address: hofmann@mathematik.th-darmstadt.de 